\title{
Ethylmalonic encephalopathy
}

INSERM

\section{Source}

INSERM. (1999). Orphanet: an online rare disease and orphan drug data base.

Ethylmalonic encephalopathy. ORPHA:51188

Ethylmalonic acid encephalopathy (EE) is defined by elevated excretion of ethylmalonic acid (EMA) with recurrent petechiae, orthostatic acrocyanosis and chronic diarrhoea associated with neurodevelopmental delay, psychomotor regression and hypotonia with brain magnetic resonance imaging (MRI) abnormalities. 